\title{
Optimized Operation of cascade reservoirs Considering Complementary Characteristics between wind and photovoltaic based on variational auto-encoder
}

\author{
Hongxuan Zhanga, Wei Hua, Rui Yub, Maolin Tangb, Lijie Dingc \\ ${ }^{1}$ Department of Electrical Engineering, Tsinghua University, Beijing 100084, China \\ ${ }^{2}$ Southwest Branch, State Grid Corporation of China, Chengdu 640041, China \\ ${ }^{3}$ SiChuan Electric Power Research Institute, State Grid Corporation of China, Chengdu 640041, China
}

\begin{abstract}
The strong variations and uncertainties of high-penetration variable renewable energy sources (RESs) has greatly challenged the operation of power systems. Considering the rapid adjustability of hydropower to complement various RESs, in this paper, a coordinated optimization model is proposed for hydro-wind-solar integrated systems. Based on the variational auto-encoder, a scenarios generation method is proposed to capture the spatial-temporal complementary and fluctuant characteristics of wind and PV power with high model accuracy and low computational complexity. The detailed hydraulic and electrical relationship between cascade reservoirs are established to make full use of the controllable regulation capacity of cascade hydropower stations. With the linearization of the head-sensitive power generation and nonlinear water head, the optimization model is reformulated into a tractable mixed integer linear programming (MILP) formulation. A basin in Northwest China integrated with prolific wind and solar resources is selected for a case study. The computational experiments on actual data demonstrate the applicability of the proposed method.
\end{abstract}

\section{RODUCTION}

THE deficiency of fossil fuels and deterioration of environment situation all over the world promotes the development of renewable energy sources(RESs) generations. The efficient scheduling and management of RES is of significant importance for the operation of modern power system. However, the increasing amount of RESs including wind and photovoltaic (PV) greatly threatens the safety and stability of power grids. On the other hand, hydroelectric sources are able to smooth these mutable RESs generations with abundant capacity and fast adjustable ability. And a growing number of researchers are gradually paying more attention to the joint operation of multi-energy hybrid systems such as hydro-wind-solar hybrid system [1]. The optimal operation of multi-energy coupling system considering complementary characteristics has become the trend of current research.

Previous studies revealed that total power generation of various renewable energy power sources might became smoother in several areas of China [2-3]. Meanwhile, with the strong regulation capacity from rich water resources and large-scale hydroelectric systems, the joint optimization of power system with solar, wind and hydropower was becoming a hot topic in China. In Longyangxia, solar-hydroelectric hybrid system had been practically put into operation in 2013, and the hydropower stations is utilized to track the fluctuation of PV [4]. [5] analysed the complementary characteristics of photovoltaic and hydropower over different time step and proposed the methods for capacity planning. Power output uncertainty was the major obstacles for the penetration of high proportional RES generations. Many works showed the advantages of combining multiple power sources. In addition, the accurately modeling of the RES considering their uncertainties is vital for the optimal operation of multi-energy hybrid systems. Scenarios based methodology is widely used to describe these uncertain resources. With a set of typical generation scenarios, the stochastic economic dispatch problems can be solved and optimal operation strategies can be determined [6]. Many works have focused on the model-based methods for scenarios production [7-9]. [7] used the empirical cumulative distribution function to model the wind power generation, while in [8], copula function is utilized to model the joint distribution of multiple renewable energy power output. [9] proposed a dynamic factor model for the estimation of the correlation between load and wind power. Nevertheless, due to the nonlinear power conversion process, spatial-temporal interactions and variant weather conditions, model-based scenarios generation methodology is difficult for practical use with the shortcomings in complexity and generalization ability. Recently, machine learning and deep learning algorithms are applied widespread in the industry such as computer vision (CV) and natural language processing (NLP). These algorithms were used for scenarios production as well, 
such as Radial Basis Functional Neural Networks (RBFNN) [10] and deep neural network (DNN) models [11]. Most of the time, these machine learning algorithms can better capture the nonlinear correlations between various RES. However, the features extraction and the acquisition of tagged data are still challenging.

Currently, there are still few studies related to the optimal coordination of multi-energy hybrid systems including wind, PV as well as hydro resources, particularly in a river basin, where cascade reservoirs can provide strong adjustable capacity. Furthermore, the detailed model of multiple RES and the quantified benefits of their complementarity still need further researches. In this paper, a coordinated optimization model is proposed for the operation of cascade hydro-wind-solar integrated system in a basin. Based on the variational auto-encoder (VAE), this paper proposes a scenarios generation method to capture the spatial-temporal complementary and fluctuant characteristics of wind and PV power with high model accuracy and low computational complexity. The detailed hydraulic and electrical relationship between cascade reservoirs are established to make full use of the controllable regulation capacity of hydropower stations. With the linearization of head-sensitive power generation and nonlinear water head, the optimization model is reformulated into a tractable mixed integer linear programming (MILP) formulation. Finally, the performance and effectiveness of proposed methods are demonstrated in the actual system.

\section{SHORT-TERM OPTIMAL OPERATION OF WIND- SOLAR-HYDRO HYBRID SYSTEM}

In this section, we propose the formulation for the shortterm optimal operation of hydro-wind-solar integrated system. Considering the spatial and temporal correlations between the wind and PV power, the joint distribution of them is obtained based on the VAE models. The refined model of cascade hydropower stations is established taking the impacts of diversified factors into account to further mitigate the variability of RESs with regulation capacity. Several methods are used for the linearization of the proposed optimization model as well.

\subsection{Variational auto-encoder for scenarios generation}

At present, VAE is one of the most commonly used generative models. The goal of the generative model is to build the target data $X$ based on the hidden variable $Z$. Usually, we assume that $Z$ obeys some given distributions (e.g. normal distribution or uniform distribution), and then train a model to map the original probability distribution of $Z$ to the training sets. Because we cannot determine the most appropriate distribution of training data, we transform the probability distribution of $\mathrm{X}$ as follow:

$$
p(X)=\int p(X \mid Z) p(Z) d Z
$$

Where $p(X)$ and $p(Z)$ represent the probability distribution of $X$ and $Z$ respectively.
Assuming that $Z$ is the standard normal distribution, we can sample from it to get the set of $Z$, and generate the scenarios based on the sample set. However, to make sure the one-to-one match of sample $X_{k}$ and target $Z_{k}$, we assume the $p\left(Z \mid X_{k}\right)$ is the exclusive distribution of $X_{k}$ and $p\left(Z \mid X_{k}\right)$ obeys the normal distribution [12]:

$$
\log p\left(Z \mid X_{k}\right)=\log N\left(Z ; u_{k}, \sigma_{k}^{2} I\right)
$$

Where $\mu_{k}$ is the mean and $\sigma_{k}^{2}$ is the variance, $I$ is the identity matrix.

To fit two parameters of normal distribution, we construct two corresponding neural network to deal with the nonlinear characteristics. In this paper, we choose to fit $\log \left(\sigma_{k}^{2}\right)$ rather than $\sigma_{k}^{2}$ because of the selection of the activation function.

With above assumptions, the probability distribution of $Z$ will obey the standard normal distribution:

$$
p(Z)=\int N(0, \mathrm{I}) p(X) d X=N(0, \mathrm{I})
$$

Thus it is practical to sample from the standard normal distribution directly for scenarios generation. On the other hand, in order to make $p\left(Z \mid X_{k}\right)$ approximate the normal distribution, we add the regularization terms into the loss function based on Kullback-leibler (K-L) divergence:

$$
L_{\mu, \sigma^{2}}=\frac{1}{2} \sum_{k=1}^{d}\left(u_{k}^{2}+\sigma_{k}^{2}-\log \sigma_{k}^{2}-1\right)
$$

Where $d$ is the dimension of $Z$. The target of K-L divergence is to get a mean of 0 and a variance of 1 to keep the accuracy and robustness of the scenarios generation model. Moreover, the mean and variance should be optimized in the back propagation process, but the sampling process is not derivable. So we sample from the standard normal distribution and obtain the derivative intermediate results with the mean and variance.

Activation function is also crucial for the performance of neural network. Rectified Linear Unit (ReLU) is the most widely used activation function with the form:

$$
\operatorname{Re} L U\left(x_{i}\right)=\max \left(0, x_{i}\right)
$$

Where $x_{i}$ is the result of $i$-th node before activation. ReLU can prevent the vanishing and exploding gradients, but some units may not be activated all the time, causing the neural network to be hard for training [13]. Therefore, we choose scaled exponential linear units (SELU) for activation. The formula of SELU is given as:

$$
\operatorname{SELU}\left(x_{i}\right)=\lambda \begin{cases}x_{i} & \text { if } x_{i}>0 \\ \alpha e^{x_{i}}-\alpha & \text { if } x_{i} \leq 0\end{cases}
$$

Where $\lambda$ and $\alpha$ are tunable parameters. [14] showed that the outputs of a fully-connected neural network would approximate the standard normal distribution when $\lambda \approx 1.058$ and $\alpha \approx 1.673$, which is suitable for the application of our model.

The structure of proposed VAE is shown in Fig.1. The Input size and output size is set as $24 \times 24$ for the convolution layer. The activation function for convolution layers and fully-connected layers are ReLU and SELU respectively. In addition, we choose the same padding mode. The "Lambda" mode is used to transform the 
sampling results from standard normal distribution based on obtained mean and variance. During the parameters tuning, it is found the VAE reaches its best performance when the dimension of mean and variance is 6 . Finally, we can use the second half of the trained model for scenarios generation.

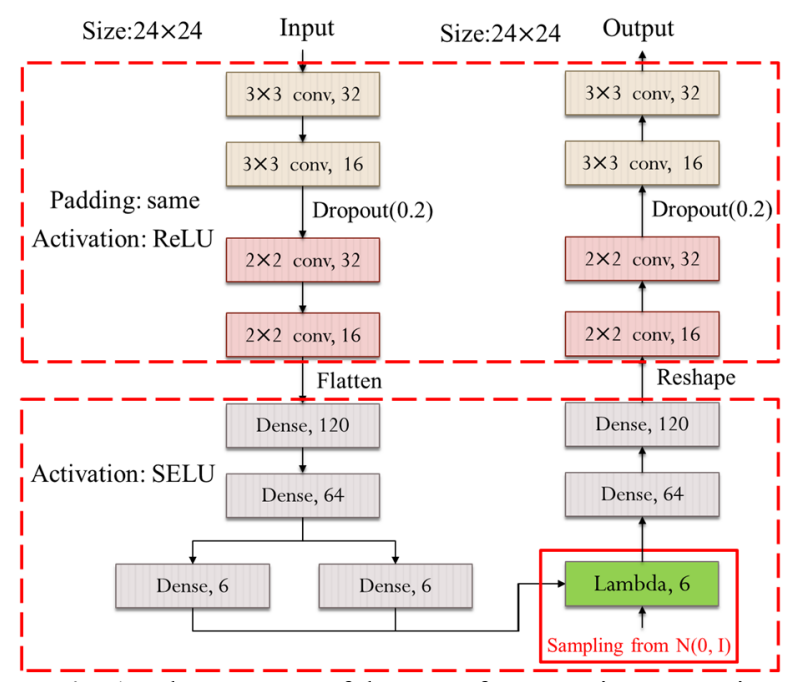

Fig. 1. The structure of the $\overline{\mathrm{V}} \overline{\mathrm{AE}}$ for scenarios generation.

\subsection{Cascade Hydropower Stations}

For the determination of optimal operation strategy for the integrated hydro-wind-solar hybrid system, the key point is the model of cascade hydroelectric plants. With nonlinear factors in many aspects, the optimization model including cascade hydropower stations is usually highdimensional, non-convex and nonlinear. First, the goal is set to maximize the generating efficiency. Because the maximum efficiency is usually attained with the highest head, the objective function is defined as follow:

$$
\max \sum_{t \in T} \sum_{i \in H} h_{i}(t)
$$

Where $h_{i}(t)$ represents the water head of $i$-th hydropower plant at time $t, H=\left\{H_{1}, H_{2}, \ldots, H_{n}\right\}$ index the $n$ cascade hydroelectric plants while $T=\left\{T_{1}, T_{2}, \ldots, T_{m}\right\}$ indicate the $m$ time periods, in this paper, $m=24$.

The operational benefit of hydropower stations is determined by dynamic characteristic curves to a great extent. Given the water discharge, water head and the conversion efficiency, we can get the power generation based on the input-output curves as follow:

$$
P_{h, i}(t)=\rho g \eta\left(h_{i}(t), q_{i}(t)\right) h_{i}(t) q_{i}(t)
$$

Where $P_{h, i}(t)$ represents the power generation of $i$-th hydropower electric plant at time $t, \rho$ and $g$ are the water density and gravitational acceleration respectively, $q_{i}(t)$ is the power generating flow of $i$-th hydropower electric plant at time $t, \eta\left(h_{i}(t), q_{i}(t)\right)$ is the generating efficiency for $i$-th hydropower electric plant with $h_{i}(t)$ and $q_{i}(t)$

Furthermore, the head, power generation, water discharge and the reservoir volume must meet the minimum and maximum constraints.

$$
\begin{gathered}
P_{i}^{m} \leq P_{h, i}(t) \leq P_{i}^{M} \\
q_{i}^{m} \leq q_{i}(t) \leq q_{i}^{M} \\
h_{i}^{m} \leq h_{i}(t) \leq h_{i}^{M} \\
V_{i}^{m} \leq V_{i}(t) \leq V_{i}^{M}
\end{gathered}
$$

Where $P_{i}^{m}$ and $P_{i}^{M}$ are the minimum and maximum value of $P_{h, i}(t)$ for $i$-th hydropower electric plant, $q_{i}^{m}$ and $q_{i}^{M}$ represent the upper and lower bounds of $q_{i}(t)$ of $i$-th hydropower electric plant, $h_{i}^{m}$ and $h_{i}^{M}$ restrict the range of $h_{i}(t)$, while $V_{i}(t)$ is limited from $V_{i}^{m}$ to $V_{i}^{M}$.

Meanwhile, many nonlinear correlations should be considered. For instance, the forebay elevation of a reservoir is nonlinearly related to the volume, while the tailwater elevation will be affected by water flow from upstream reservoir. Therefore, the formula of head is as follow:

$$
\begin{gathered}
Z_{i}^{u}(t)=\mathbb{C}_{i}\left(V_{i}(t)\right) \\
Z_{i}^{d}(t)=\mathbb{R}_{i}\left(q_{i}(t)+\sigma_{i}(t)\right) \\
h_{i}(t)=\frac{1}{2}\left[Z_{i}^{u}(t)+Z_{i}^{u}(t-1)\right]-Z_{i}^{d}(t)-h_{i}^{\text {loss }}(t)
\end{gathered}
$$

Where $\mathbb{C}_{i}(\cdot)$ represents the forebay elevationreservoir volume curves while $\mathbb{R}_{i}(\cdot)$ means the tailwater elevation-water flow curves; $Z_{i}^{u}(t)$ is the upper water level at time $t$ for $i$-th hydropower plant, $V_{i}(t)$ is the reservoir volume of $i$-th hydropower plant at time $t$; $Z_{i}^{d}(t)$ is the tail water level at time $t$ for $i$-th hydropower plant, $\sigma_{i}(t)$ is the water spillage of $i$-th hydropower plant at time $t ; h_{i}^{\text {loss }}(t)$ is the water head loss at time $t$ for $i$-th hydropower plant. In order to simplify the formulation of water head, we assume the head is linearly related to the forebay elevation and tail water level, and the water head loss is constant.

Also, the water relations between different cascade reservoirs should be taken into account. Thus we get hydraulic continuity equations as below:

$$
\begin{aligned}
V_{i}(t+1)= & V_{i}(t)+Q_{i}(t)+q_{i-1}\left(t-\tau_{i-1}\right) \\
& +\sigma_{i-1}\left(t-\tau_{i-1}\right)-q_{i}(t)-\sigma_{i}(t)
\end{aligned}
$$

Where $\tau_{i-1}$ indicates the water time delay from $i$-1-th hydropower plant to $i$-th hydropower plant; $q_{i-1}\left(t-\tau_{i-1}\right)$ and $\sigma_{i-1}\left(t-\tau_{i-1}\right)$ means the effects of the upstream hydropower station on the downstream hydropower station through the water generating flow and water spillage. $Q_{i}(t)$ is the runoff of $i$-th hydropower plant at time $t$. 


\subsection{Solutions of the optimization model}

Although a refined model for hydro-wind-solar hybrid system is established, the proposed optimization is difficult to solve because of nonlinear factors. There is still no general and perfect algorithm to deal with highdimensional, non-convex and non-linear problems. Although some heuristic algorithms, such as genetic algorithm and particle swarm optimization, can be used to solve such problems, the results are usually not optimal and the convergence process is slow. In this paper, we make full use of several linearization methods for the (7), (12), (13), and transform this model into a mixed integer linear programming (MILP) problem, which can be solved by mature commercial software, such as CPLEX and Gurobi.

To deal with the nonlinear water head, we use piecewise linearization method to linearize the forebay elevation-reservoir volume curves and tailwater elevationwater flow curves. Given $\chi=1,2, \ldots U$ intervals for upper stream water level and $\gamma=1,2, \ldots D$ for tail water level, we obtain:

$$
\begin{aligned}
& Z_{i}^{u}(t)=\sum_{\chi=1}^{U} \delta_{i}^{\chi}(t)\left[\alpha_{i}^{\chi} \theta_{i}^{\chi}(t)+\beta_{i}^{\chi}\right] \\
& Z_{i}^{d}(t)=\sum_{\gamma=1}^{D} \delta_{i}^{\gamma}(t)\left[\alpha_{i}^{\gamma} \theta_{i}^{\gamma}(t)+\beta_{i}^{\gamma}\right]
\end{aligned}
$$

Where $\alpha_{i}^{\chi}$ and $\beta_{i}^{\chi}$ are the linearization coefficients of $Z_{i}^{u}(t)$ in interval $\chi$, while $\alpha_{i}^{\gamma}$ and $\beta_{i}^{\gamma}$ are the linearization coefficients of $Z_{i}^{d}(t)$ in interval $\gamma . \theta_{i}^{\chi}(t)$ and $\theta_{i}^{\gamma}(t)$ indicate the scope of reservoir volume and water flow in interval $\chi$ and $\gamma$ separately. $\delta_{i}^{\chi}(t)$ and $\delta_{i}^{\gamma}(t)$ are binary variables to give the intervals $Z_{i}^{u}(t)$ and $Z_{i}^{d}(t)$ belong to. In addition, $\delta_{i}^{\chi}(t)$ and $\delta_{i}^{\gamma}(t)$ need to satisfy following constraints:

$$
\begin{aligned}
& \sum_{\chi=1}^{U} \delta_{i}^{\chi}(t)=1 \\
& \delta_{i}^{\chi}(t) \in\{0,1\} \\
& \sum_{\gamma=1}^{D} \delta_{i}^{\gamma}(t)=1 \\
& \delta_{i}^{\gamma}(t) \in\{0,1\}
\end{aligned}
$$

Thus we can get:

$$
\begin{gathered}
V_{i}(t)=\sum_{\chi=1}^{U} \delta_{i}^{\chi}(t) \theta_{i}^{\chi}(t) \\
q_{i}(t)+\sigma_{i}(t)=\sum_{\gamma=1}^{D} \delta_{i}^{\gamma}(t) \theta_{i}^{\gamma}(t)
\end{gathered}
$$

Accordingly, we get the linearized water head with above assumptions. Moreover, the volume of reservoir with large capacity usually remains nearly constant in the day-ahead time scale, so the formulation can be directly linearized around the current head level, which can meet the accuracy requirements with greatly reduced number of binary variables.

For the nonlinear input-output curves, we assume that the dynamic characteristics of each hydro unit in the same hydropower station are consistent. Besides, the reservoir volume and runoff will not change drastically in a day, we can make the hypothesis that the power generation efficiency is constant. Nonetheless, the power generation characteristic of hydropower stations is still a non-convex bilinear function. To this regard, we use McCormick's inequalities to build the convex envelope for dynamic curves of hydropower units and transform the model into a tractable one [15]. The computational errors are determined by the relaxation intervals. So we are able to further reduce the search area by increasing the binary variables to restrict the range of continuous variables, but the computational complexity is supposed to be considered. The detailed process can be found in [16].

Based on the obtained scenarios, the power generation of wind or PV can be seen as certain variable in a particular scenario. Then, the key point is the control of cascade reservoirs. To sum up, the complete procedure is as seen in Fig 2.

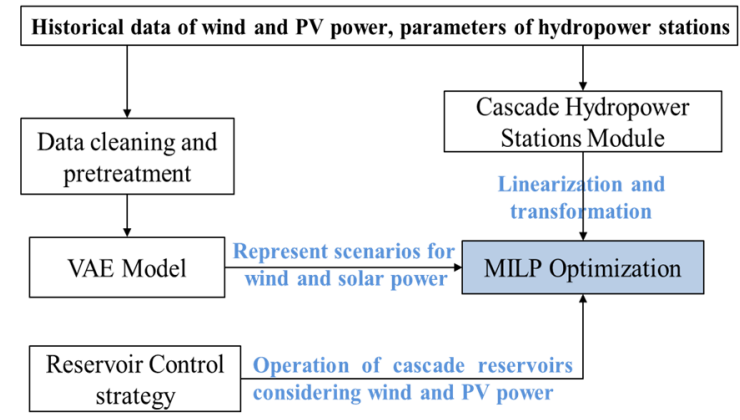

Fig. 2. The complete procedure for proposed coordinated optimization model.

\section{ReSULTS AND DISCUSSION}

In this section, we will illustrate the performance of our methods. First, the generated scenarios for wind and PV power considering spatial and temporal correlations are compared with real historical data, proving the efficiency of VAE model. Next the MILP problems for hydro-windsolar hybrid system is solved to get the optimal operation strategy considering complementary coordination.

\subsection{Data Description}

The training and validation dataset for wind and photovoltaic power is built based on the actual data from a province in northwest China. Five cascade hydropower stations system including 4 adjustable hydropower stations and 1 run-off hydroelectric plant is chosen for analysis. The power output of 6 wind farms and 18 solar arrays all the year round is utilized. By resampling the original data, the input data has resolution of 1 hour. Thus we can form the $24 \times 24$ input size without zero padding. To reflect the advantage of power fluctuation and nonlinear correlations handling, we use the VAE to directly fit the power generation. 


\subsection{Scenarios Generation}

In this paper, the VAE network is established based on Keras 2.2.2 and Tensorflow 1.10.0. The Adam optimizer is selected
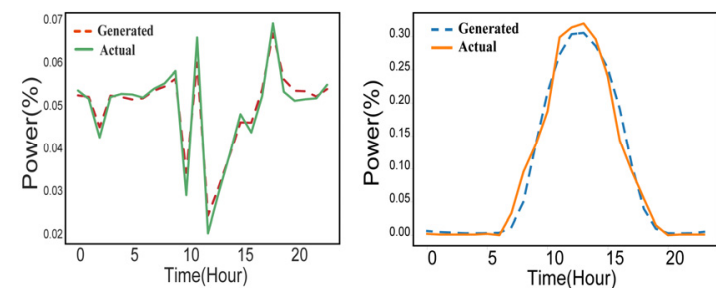

Fig. 3. Daily wind and photovoltaic power output comparison between actual data and generated scenarios.

for back propagation. A laptop with four $1.80 \mathrm{GHz}$ CPUs is used for training. The detailed parameters and structure of proposed VAE is seen in Fig 1. We use ReLU as the activation function of convolutional layer, SELU as the activation function of fully-connected layer and Sigmoid for the output layer. Then we can intercept the decoder part as the generative model if the VAE is completely trained. Some generated scenarios are illustrated to verify the validity of our proposed method as seen in the Fig 3 .

Although the power output in the test samples is not trained, the generative model automatically produces some scenarios close to the test samples as Fig 3 shows. The fluctuation of wind and PV power are accurately fitted. The proposed VAE structure can not only reproduce the data in the training set, but also create new but practical scenarios based on the learned

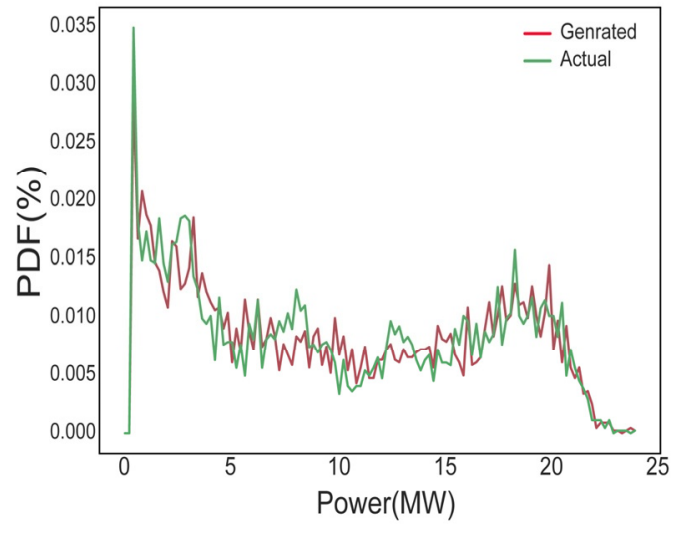

Fig. 4. Probability distribution of actual samples and generated scenarios for solar power.

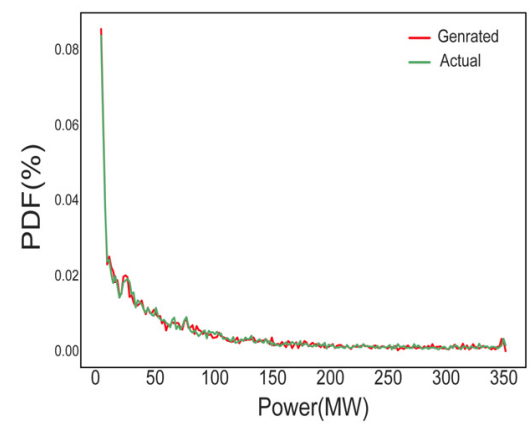

Fig. 5. Probability distribution of actual samples and generated scenarios for wind power. features. The results demonstrate that the scenarios generated by VAE model is consistent with the actual situation

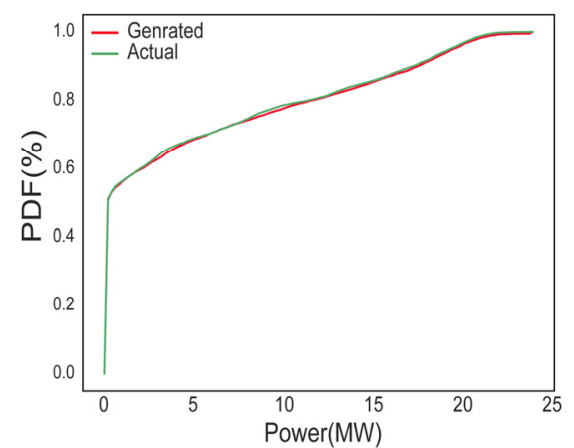

Fig. 6. Cumulative distribution of actual samples and generated scenarios for wind power.

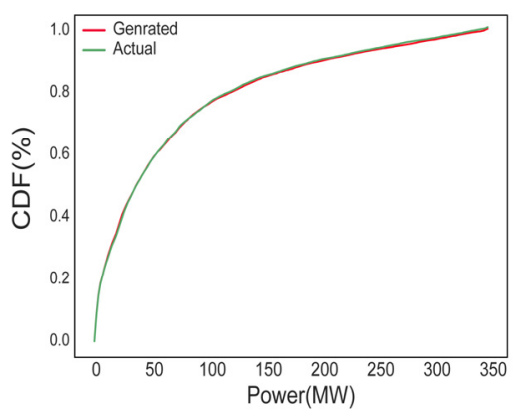

Fig. 7. Cumulative distribution of actual samples and generated scenarios for wind power.

On the other hand, we can analyze the statistical characteristics of generated scenarios in the long term. The probability distribution functions (PDF) of actual power generation data and generated scenarios for wind and PV are displayed in Fig 4 and Fig 5. And the cumulative distributions function (CDF) of actual power generation data and generated scenarios are shown in Fig 6 and Fig 7. Among them, the time periods when the power output is zero are removed for probability distribution of $\mathrm{PV}$ power to compare the results in better visualization.

As seen from Fig 4 to Fig 7, the probability distribution and cumulative distribution of generated scenarios accurately fit the historical samples, which indicates that the VAE model is able to learn the distribution characteristics of training samples. Based on the unsupervised training theory, we achieve the performance of probability modeling of traditional methods.

Furthermore, in order to estimate the spatial correlation of generated scenarios, we calculate the correlation coefficients between historical data and generated scenarios for wind and PV power respectively as seen in Fig 8 .

As shown in Fig 8, although the spatial correlation coefficients between some wind farms and solar arrays are slightly different for actual data and generated data, the VAE model can capture the spatial correlation characteristics as a whole. It is demonstrated that VAE model can capture both the short-term characteristics and long-term characteristics of wind and PV power, such as frequent fluctuation, peak-valley changes, PDF, CDF, and spatial correlations. The generated scenarios almost reproduce the characteristics 
of actual data without modifying the VAE network structure. Compared to the traditional approach, proposed generative model can be more representative of the actual operation characteristics of the wind farms and solar arrays.
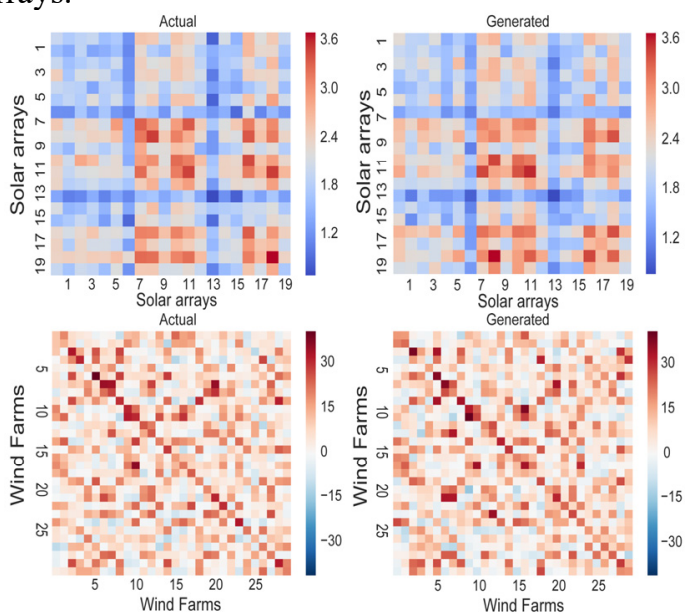

Fig. 8. Spatial correlation coefficients matrix for one-day training data and generated scenarios of wind and PV power.

\subsection{Optimal Operation of Hydro-wind-solar Hybrid System}

Based on the obtained scenarios for wind and solar generation, we can analysis the operation of hydro-windsolar hybrid system in the given power transmission demand. The wind and PV power output are definitive in a certain scenario. Therefore, the target is to balance the remaining load through the regulation of cascade hydropower stations.

In our model, in order to get the maximum generating head and system operation efficiency, the five cascade hydropower stations should be operated appropriately. The first and second reservoirs should undertake the base load in the maximum generating efficiency and control the water discharge to the downstream considering the characteristics of wind and PV. Next, the third and fourth reservoir is supposed to mainly track the fast fluctuation of wind and solar power. The last hydropower station should provide stable power support in the peak-load hours. Then we run the proposed optimal operation

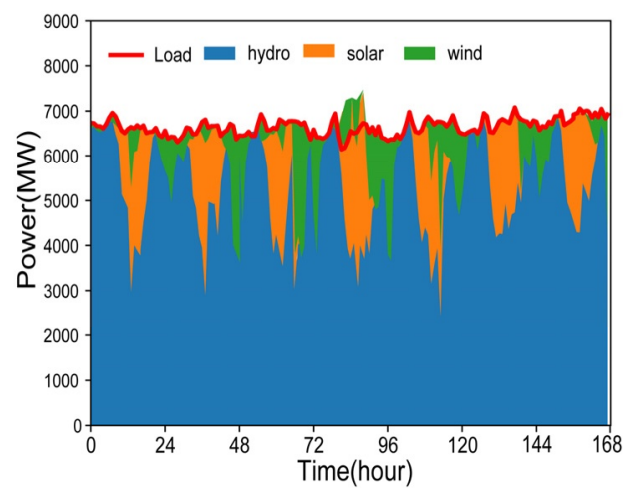

Fig. 8. Weekly optimal coordinated operation of hydrowind-solar hybrid system. power output from cascade hydropower stations, wind farms and solar arrays separately over a week. The load curve is also displayed in this graph. The result proves that the power generation of only hydro-wind-solar hybrid system can meet the given load demand most of the time. During the simulation time scale, renewable energy curtailment occurs only on the fourth day because of large amount of wind-PV power generation and limited adjustable ability from hydropower.

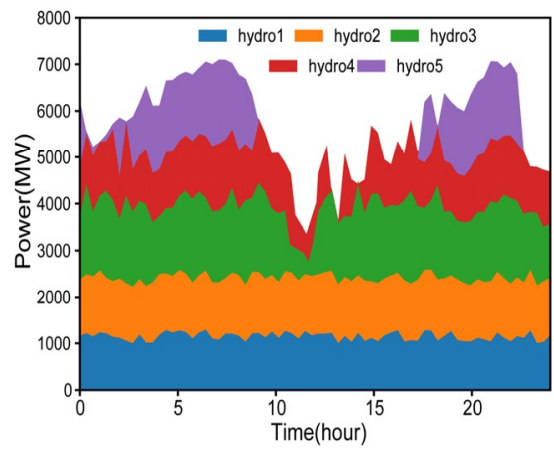

Fig. 9. Power output of five cascade hydropower stations in a typical day.

Typically, the operation of five cascade hydropower stations on a certain day is described in Fig 9. Although the power output of the first two cascade hydropower stations is influenced by inflow, it is relatively stable to provide the base load. With large regulation capacity and fast adjustable ability, the third and fourth cascade hydropower stations can balance the fluctuation of wind and PV power around $12 \mathrm{~h}$. The fifth hydropower station is capable of undertaking partial load in the peak hour and impound water for the rest of time because of the constraints of other factors. Finally, with the full use of the regulation capacity from cascade hydropower stations, the optimal operation of only hydro-wind-solar hybrid system achieves a good performance.

\section{CONCLUSION AND FutURE WORK}

In this paper, we propose a VAE based model for scenarios generation considering the spatial and temporal correlations between wind and PV power. The coordinated operation strategy is made to fully utilize the regulation capacity of cascade reservoir. By utilizing the VAE network, the results indicate that VAE structure can accurately capture the short-term and long-term characteristics and correlations between wind and PV power. By solving the MILP model, we find that the power output of only hydro-wind-solar hybrid system can meet the target load demand most of the time, which is capable of reducing computational complexity of latter scheduling and decreasing the renewable energy curtailment.

In future research, the structure of VAE network and loss function can be further optimized. On the other hand, the operation of hydro-wind-solar hybrid system will be analyzed in drought and flood season. The uncertain load variation and electricity market may be considered as well. 


\section{ACKNOWLEDGMENT}

This work was supported by Science and Technology Project of the State Grid Sichuan Electric Power Company (521999180002).

\section{REFERENCES}

1. Ming B, Liu P, Guo S, et al. Robust hydroelectric unit commitment considering integration of large-scale photovoltaic power: A case study in China[J]. Applied Energy, 2018, 228.

2. Greve Z D. Robust optimisation for hydroelectric system under uncertainty[J]. Power Systems IEEE Transactions on, 2018.

3. Ming B, Liu P, Cheng L, et al. Optimal daily generation scheduling of large hydro-photovoltaic hybrid power plants[J]. Energy Conversion \& Management, 2018, 171:528-540.

4. Lin L, Li L, Jia L. An optimal capacity configuration method of wind/PV and energy storage cogeneration system[C]// Pes General Meeting | Conference \& Exposition. IEEE, 2014:1-5.

5. X. Wang, Y. Mei, Y. Kong, Y. Lin, and H. Wang, "Improved multi-objective model and analysis of the coordinated operation of a hydro-wind-photovoltaic system," ENERGY, vol. 134, pp. 813-839, 2017.

6. Chen Y, Wang Y, Kirschen D S, et al. Model-Free Renewable Scenario Generation Using Generative Adversarial Networks[J]. IEEE Transactions on Power Systems, 2017, PP(99):1-1.

7. Ma X Y, Sun Y Z, Fang H L. Scenario Generation of Wind Power Based on Statistical Uncertainty and Variability[J]. IEEE Transactions on Sustainable Energy, 2013, 4(4):894-904.

8. Molnar P, Steinle Camargo L A, Soares Ramos D. Applying copulas functions for wind and hydro complementarity evaluation: A Brazilian case[C]// European Energy Market. IEEE, 2015:1-6.

9. Lee D, Baldick R. Load and Wind Power Scenario Generation Through the Generalized Dynamic Factor Model[J]. IEEE Transactions on Power Systems, 2016, 32(1):400-410.

10. Sideratos G, Hatziargyriou N D. Probabilistic Wind Power Forecasting Using Radial Basis Function Neural Networks[J]. IEEE Transactions on Power Systems, 2012, 27(4):1788-1796.

11. Vagropoulos S I, Kardakos E G, Simoglou C K, et al. ANN-based scenario generation methodology for stochastic variables of electric power systems[J]. Electric Power Systems Research, 2016, 134(29 Suppl 2):9-18.

12. Dahl G E, Sainath T N, Hinton G E. Improving deep neural networks for LVCSR using rectified linear units and dropout[C]// IEEE International Conference on Acoustics, Speech and Signal Processing. IEEE, 2013:8609-8613.
13. Klambauer G, Unterthiner T, Mayr A, et al. SelfNormalizing Neural Networks[J]. 2017.

14. Chen K, Chen K, Wang Q, et al. Short-term Load Forecasting with Deep Residual Networks[J]. IEEE Transactions on Smart Grid, 2018, PP(99):1-1.

15. Al-Khayyal F A, Falk J E. Jointly Constrained Biconvex Programming[J]. Mathematics of Operations Research, 1983, 8(2):273-286.

16. Lima R M, Marcovecchio M G, Novais A Q, et al. On the Computational Studies of Deterministic Global Optimization of Head Dependent Short-Term Hydro Scheduling[J]. IEEE Transactions on Power Systems, 2013, 28(4):4336-4347. 